\title{
Efficacy of quercetin against chemically induced murine oral squamous cell carcinoma
}

\author{
DANIEL DROGUETT ${ }^{1,2}$, CHRISTIAN CASTILLO ${ }^{2}$, ELBA LEIVA $^{3}$, CRISTINA THEODULOZ $^{4}$, \\ GUILLERMO SCHMEDA-HIRSCHMANN ${ }^{4 *}$ and ULRIKE KEMMERLING ${ }^{2 *}$
}

\author{
${ }^{1}$ Department of Stomatology, Faculty of Health Sciences, University of Talca, Talca 3460000; \\ ${ }^{2}$ Program of Anatomy and Developmental Biology, Institute of Biomedical Sciences, Faculty of Medicine, \\ University of Chile, Santiago 8380453; ${ }^{3}$ Department of Clinical Biochemistry and Immunohematology, \\ Faculty of Health Sciences; ${ }^{4}$ Institute of Chemistry of Natural Resources, University of Talca, Talca 3460000 , Chile
}

Received September 3, 2014; Accepted June 22, 2015

DOI: $10.3892 / \mathrm{ol} .2015 .3598$

\begin{abstract}
Oral squamous cell carcinoma (OSCC) is the most common form of head and neck cancer, and oxidative damage is associated with the development of OSCCs. Antioxidants have therefore been proposed for use as chemoprotective agents against different types of cancer. In the present study, the effect of the antioxidant quercetin, administered at doses of 10 and $100 \mathrm{mg} / \mathrm{kg} / \mathrm{day}$, was investigated in an experimental murine model of 4-nitroquinoline 1-oxide (4-NQO)-induced carcinogenesis. The survival of the treated animals, the plasmatic levels of reduced glutathione and the type and severity of lesions (according the International Histological Classification of Tumors and Bryne's Multifactorial Grading System for the Invasive Tumor Front) were assessed. Additionally, the organization of the extracellular matrix was analyzed by carbohydrate and collagen histochemistry, and immunohistochemistry was used to assess the expression of the tumor markers proliferating cell nuclear antigen and mutated p53. The results indicate that, despite the promising effect of quercetin in other studies, this drug is ineffective as a chemoprotective agent against 4-NQO-induced OSCC in mice at the assayed doses.
\end{abstract}

\section{Introduction}

Oral squamous cell carcinoma (OSCC) is the most common form of head and neck cancer, accounting for $\sim 3 \%$ of malignancies worldwide and 500,000 newly diagnosed cases annually.

Correspondence to: Dr Ulrike Kemmerling, Program of Anatomy and Developmental Biology, Institute of Biomedical Sciences, Faculty of Medicine, University of Chile, 1027 Independencia, Block A, Santiago 8380453, Chile

E-mail: ukemmerling@med.uchile.cl

${ }^{*}$ Contributed equally

Key words: chemoprevention, oral squamous cell carcinoma, quercetin, 4-nitroquinoline 1-oxide
The mechanisms underlying the development of OSCC have not been fully elucidated; however, tobacco and alcohol use are reported to increase the risk for developing this type of cancer (1-3). Additionally, despite the accessibility of the oral cavity for medical examination, the majority of cases of oral cancer are only detected at advanced stages; this is one of the reasons for the low OSCC survival rates (4), which rarely exceed $50 \%$ (5).

Surgery and chemotherapeutic agents, including ifosfamide, 5-fluorouracil, taxane and methotrexate, constitute the primary treatment approaches for this malignancy (6). However, patients eventually succumb to the disease with the development of resistance to such agents.

Tobacco smoke produces various free radicals, reactive oxygen species (ROS) and reactive nitrogen species (RNS), leading to the production of hydrogen peroxide, superoxide and nitric oxide in cells, which causes oxidative/nitrosative damage and oxidative stress (7). Due to their ability to induce DNA damage, ROS and RNS are crucial determinants of the development of OSCC (3). Conversely, antioxidants may exert a protective effect against the molecular and cellular damage that results from the interactions of ROS and RNS $(2,8)$.

The most frequently used natural antioxidants are flavonoids, a group of polyphenolic compounds commonly found in medicinal plants, vegetables, fruits and a various beverages, including tea, coffee and wine (9). Quercetin (3,3',4',5,7-pentahydroxyflavone) is the most abundant dietary flavonoid and has been widely used for the prevention and treatment of cardiovascular diseases and cancer (10). The cancer-preventive effects of quercetin have been attributed primarily to its antioxidant activity; it is able to act as a scavenger of radicals and form complexes with metal ions and DNA (9). In addition, a number of studies have demonstrated that quercetin may be a plausible agent for overcoming multidrug resistance, a significant impediment to successful chemotherapy (11).

Numerous in vitro studies have demonstrated consistent anticancer effects of quercetin in various cell lines and tumors, including oral cavity cancer $(12,13)$. Similarly, studies investigating the chemopreventive effects of quercetin in vivo have revealed that its oral administration may inhibit the induction of carcinogenesis, particularly in the colon (14). When 
administered in the diet, quercetin is reportedly able to prevent the initiation, growth and/or dissemination of induced tumors in animal models (15). However, these results are controversial, as both a lack of an effect and inhibition of tumor growth have been reported by independent studies $(13,16)$.

Experimental carcinogenesis induced by 4-nitroquinoline 1-oxide (4-NQO) in mice is one of the most frequently used animal models for the study of oral cancer (17). In this system, the clinical, histological and molecular changes of the oral mucosa are similar to those observed in humans during oral carcinogenesis $(18,19)$.

The current study aimed to examine the effect of orally administered quercetin in 4-NQO-treated mice. The survival rate of the treated animals, plasmatic levels of reduced glutathione (GSH) as measure for systemic oxidative status, and the type and severity of lesions were assessed. In addition, the organization of the extracellular matrix (ECM) was analyzed using carbohydrate and collagen histochemistry, and the expression of the tumor markers proliferating cell nuclear antigen (PCNA) and mutated p53 were assessed using immunohistochemistry.

\section{Materials and methods}

Animals and experimental design. A total of 70 six-week-old, male, CF-1 mice, obtained from the Public Health Institute of Chile (Santiago, Chile), were maintained under controlled conditions (access to food and water ad libitum, 12/12 h light/dark cycle, $22^{\circ} \mathrm{C}$ ) in the Animal Facility of the University of Talca (Talca, Chile). The mice were randomly divided into four groups: Group 1, 4-NQO $(n=20)$; group 2, 4-NQO + quercetin $(10 \mathrm{mg} / \mathrm{kg} /$ day $)(\mathrm{n}=20)$; group $3,4-\mathrm{NQO}+$ quercetin (100 mg/kg/day) $(\mathrm{n}=20)$; and group 4, untreated controls $(n=10)$ (Fig. 1). OSCC was induced as described previously $(16,17)$. Briefly, mice were treated with a solution of $100 \mu \mathrm{g} / \mathrm{ml}$ propylene glycol/4-NQO (Sigma-Aldrich, St. Louis, MO, USA) in drinking water for 16 weeks. Quercetin (Sigma-Aldrich), at a dose of 10 or $100 \mathrm{mg} / \mathrm{kg} /$ day, was administered orally over the course of 18 weeks [1 week prior to (week 0), during, and 1 week after the 4-NQO treatment]. At week 29 (Fig. 1), the animals were sacrificed by cervical dislocation, their tongues were dissected, and their blood was collected and processed for further analysis. The survival of the animals was recorded daily.

This experimental protocol was approved by the Bioethics Committee of the University of Talca.

Histological and histochemical techniques. The tongues of the mice were processed for conventional histology. Sections were stained with hematoxylin and eosin for routine histopathological analysis, Picro Sirius Red-hematoxylin (cat no. 365548; Sigma-Aldrich) for collagen histochemistry (20), and periodic acid-Schiff (PAS) for carbohydrate-containing tissue elements (Sigma-Aldrich kit 395B). As a control for Picro Sirius Red and PAS, $5 \mu$ m-thick sections were incubated, respectively, with a solution of collagenase ( $2 \mu \mathrm{g} / \mathrm{ml}$; cat no. 10103586001; Roche, Basel, Switzerland) or $\alpha$-amylase ( $4 \mu \mathrm{g} / \mathrm{ml}$; cat no. A6380; Sigma-Aldrich) in phosphate-buffered saline (PBS; pH 6.0), for $30 \mathrm{~min}$ at $37^{\circ} \mathrm{C}$, prior to the histochemical reaction. A reduction in the intensity of the Picro Sirius Red reaction or
PAS staining following enzyme treatment was considered as evidence of the presence of carbohydrates or collagen (data not shown). In ten randomly selected fields, the intensity of the histochemical reaction was analyzed by two independent observers using polarized light microscopy (Leitz Orthoplan microscope; Leica Camera AG, Wetzlar, Germany) and the signal intensity was scored as follows: -, absent; +, weak; ++ , moderate; +++ , high (21).

Diagnosis of OSCC and pre-neoplastic lesions was performed by an oral pathologist (Mr. Daniel Droguett). The severity of the lesions was determined according to the World Health Organization (WHO) International Histological Classification of Tumors and Histological Malignancy Grading System for the Invasive Tumor Front (ITF) (22). The resulting scores were grouped by assigning each score a value of 1-3, according to the method developed by Tumuluri et al (23) and the ITF morphological characteristics were compared separately, as proposed by Bryne's Multifactorial Grading System (24).

Immunohistochemistry. Tongues were processed using standard immunoperoxidase techniques (21) to label PCNA (rabbit anti-mouse polyclonal IgG antibody; \#sc-7907; Santa Cruz Biotechnology, Inc., Dallas, TX, USA; dilution, 1:100 v/v) and mutated p53 (Novocastra ${ }^{\mathrm{TM}}$ rabbit anti-mouse polyclonal antibody (CM5); \#P53-CM5P; Leica Biosystems Nussloch GmbH, Nussloch, Germany; dilution, 1:100 v/v). The primary antibodies were applied individually to each section for $60 \mathrm{~min}$ at $37^{\circ} \mathrm{C}$. Immunostaining was performed using a horseradish peroxidase-labeled streptavidin biotin kit (R.T.U. Vectastain ${ }^{\circledR}$ Universal ABC Kit; Vector Laboratories, Inc., Burlingame, CA, USA) following the manufacturer's protocol, with diaminobenzidine as the chromogen. Sections were counterstained with Mayer's hematoxylin (ScyTek Laboratories, Inc., Logan, UT, USA) and mounted with Entellan (Merck Millipore, Darmstadt, Germany). Immunohistochemical controls were processed by substituting the primary antibodies with PBS, and all of the controls were negative. For analysis of staining, ten fields were randomly selected, the localization and intensity of the immunoreactivity was analyzed by two independent observers using light microscopy (BA310; Motic, Hong Kong, China) and the signal intensity was scored as follows: -, absent; +, weak; ++, moderate; +++, high (21).

GSH levels. At week 29 of the experimental phase, GSH levels were measured using the method described by Beutler et al (25), with 5,5'-dithiobis-(2-nitrobenzoic acid) (Sigma-Aldrich)as the sulfhydryl reagent. The optical density at $432 \mathrm{~nm}$ was measured in a spectrophotometer (Spectronic GENESYS ${ }^{\mathrm{TM}}$ 10S UV-Vis; Thermo Fisher Scientific, Waltham, MA, USA). GSH levels were determined using the molar absorption coefficient of GSH at $432 \mathrm{~nm}$ $\left(1.36 \times 10^{4} \mathrm{l} / \mathrm{mol} / \mathrm{cm}\right)$ and expressed as $\mathrm{mg} / \mathrm{dl}$.

Statistical analysis. Analysis of survival in the different groups was performed using the Kaplan-Meier method followed by comparison of the groups by log rank test. Quantitative data were expressed as the mean \pm standard deviation and analyzed using analysis of variance followed by Tukey's 


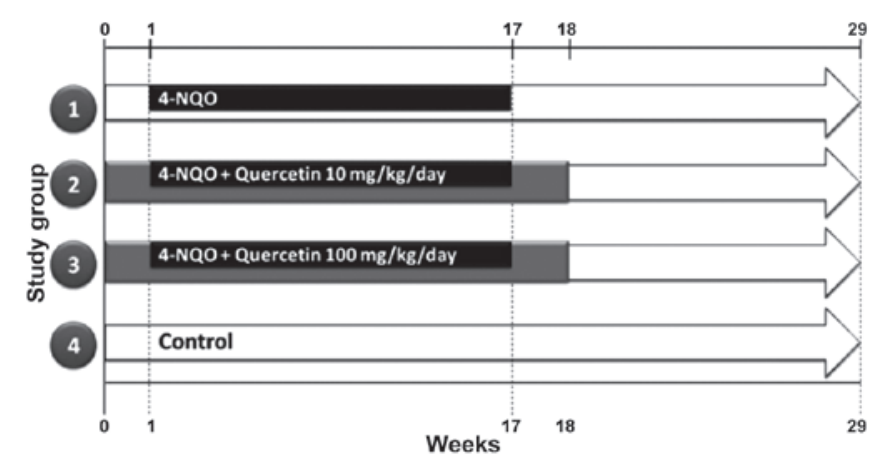

Figure 1. Experimental protocol for 4-NQO-induced carcinogenesis and quercetin treatment. A total of 70 six-week-old, male CF-1 mice were randomly divided into 4 groups: (group 1) 4-NQO (n=20); (group 2) 4-NQO + quercetin $(10 \mathrm{mg} / \mathrm{kg} /$ day $)(\mathrm{n}=0)$; (group 3$) 4-\mathrm{NQO}+$ quercetin $(100 \mathrm{mg} / \mathrm{kg} /$ day) $(n=20)$; and (group 4$)$ untreated control $(n=10)$. The mice were treated with a propylene glycol/4-NQO solution in their drinking water at a concentration of $100 \mu \mathrm{g} / \mathrm{ml}$ for 16 weeks (black bars). Quercetin was administered orally at doses of 10 or $100 \mathrm{mg} / \mathrm{kg} /$ day (grey bars) over 18 weeks in groups 2 and 3. At week 29 , the animals were sacrificed and the experimental procedures were performed. 4-NQO, 4-nitroquinoline 1-oxide.

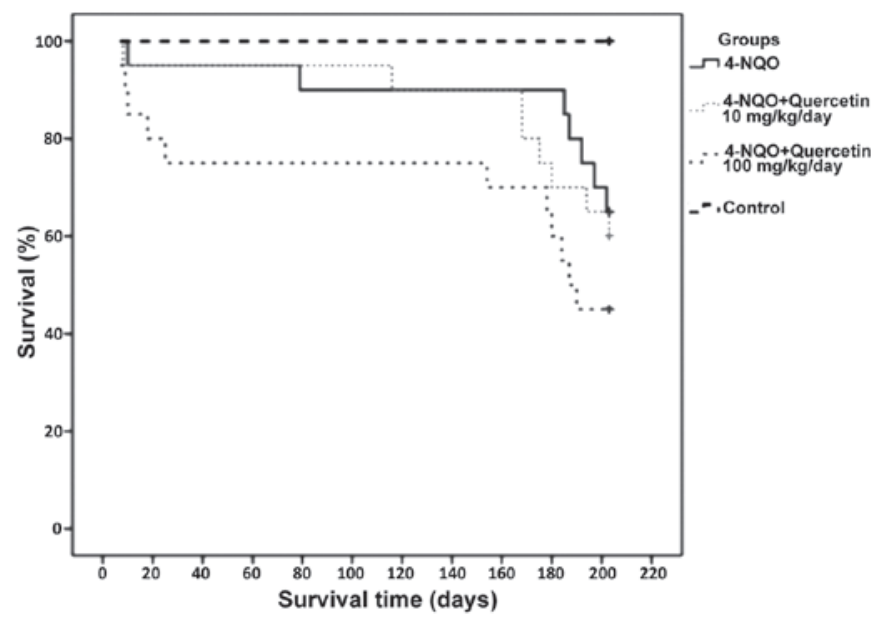

Figure 2. Quercetin does not improve survival rate in mice treated with 4-NQO. A Kaplan-Meier survival analysis of mice treated with 4-NQO to induce oral squamous cell carcinoma was conducted. No statistically significant difference was identified between the experimental groups, while statistically significant differences were observed between the three groups of 4-NQO-treated mice (with and without quercetin administration) and healthy, untreated control animals $(\mathrm{P} \leq 0.05)$. 4-NQO, 4-nitroquinoline 1-oxide.

post-test. Qualitative data were analyzed by Fisher's exact test. SPSS software (version 14.0; SPSS, Inc., Chicago, IL, USA) was used for all calculations and Prism software (version 5.0; GraphPad, San Diego, CA, USA) was used for all graphics. A statistical significance threshold of $\mathrm{P} \leq 0.05$ was used for all results.

\section{Results}

Quercetin does not improve survival in mice treated with 4-NQO. Mice treated with 4-NQO alone or in combination with 10 or $100 \mathrm{mg} / \mathrm{kg} /$ day quercetin exhibited survival rates of $65 \%(n=14), 60 \%(n=13)$ and $45 \%(n=9)$, respectively. No statistically significant difference was identified between these groups (groups 1, 2 and 3). Notably, however, the group

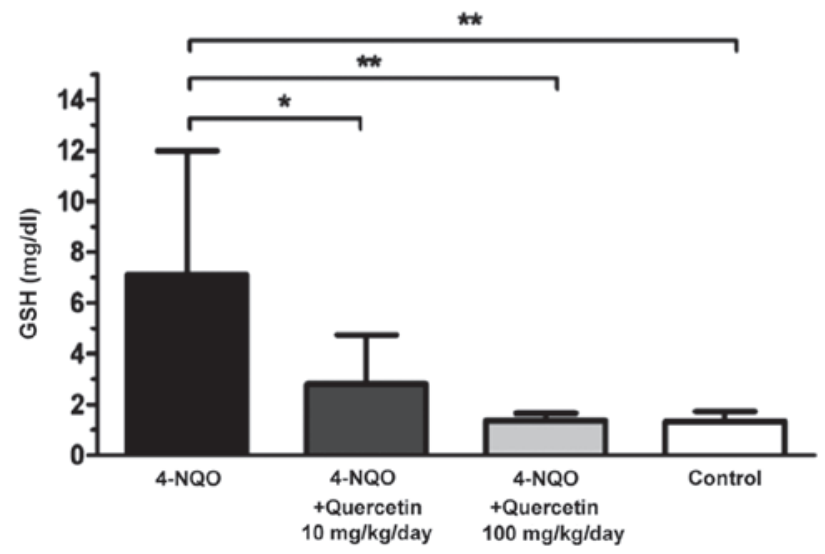

Figure 3. Quercetin decreases plasmatic GSH levels in 4-NQO-treated mice. Mice were treated with 4-NQO (black bar) alone or in combination with $10 \mathrm{mg} / \mathrm{kg} /$ day quercetin (dark gray bar) or $100 \mathrm{mg} / \mathrm{kg} /$ day quercetin (light gray bar). The white bar represents healthy, untreated control animals. GSH levels were measured at week 29 of the experimental phase. The data are expressed as the mean \pm standard deviation, and statistical analysis was performed using analysis of variance followed by Tukey's multiple comparisons test. "P $\leq 0.05$ and ${ }^{* *} \mathrm{P} \leq 0.01$. GSH, reduced glutathione; 4-NQO, 4-nitroquinoline 1-oxide.

treated with the higher dose of quercetin (100 mg/kg/day) had the poorest survival rate (45\%). The only statistically significant difference observed was between the healthy, untreated control group (group 4) and each of groups 1-3 ( $\mathrm{P} \leq 0.05)$; the animals in group 4 had a survival rate of $100 \%$ (Fig. 2).

Quercetin decreases plasmatic GSH levels in 4-NQO-treated mice. Plasmatic GSH levels were determined at the end of the experimental phase (week 29). Healthy, untreated control mice and animals treated with 4-NQO in combination with one of the two doses of quercetin exhibited significantly lower levels of plasmatic GSH compared with mice treated only with the carcinogen (Fig. 3). Mice in the 4-NQO-treated group (Fig. 3, black bar) exhibited a mean plasmatic GSH level of $7.1 \pm 4.9 \mathrm{mg} / \mathrm{dl}$, while animals treated with 4-NQO plus $10 \mathrm{mg} / \mathrm{kg} /$ day (Fig. 3, dark grey bar) or $100 \mathrm{mg} / \mathrm{kg} /$ day (Fig. 3, light grey bar) quercetin had a mean GSH level of $2.8 \pm 1.9 \mathrm{mg} / \mathrm{dl}$ ( $\mathrm{P} \leq 0.05$ vs. 4-NQO-only group) and $1.4 \pm 0.3 \mathrm{mg} / \mathrm{dl}$ ( $\mathrm{P} \leq 0.01$ vs. 4-NQO-only group), respectively. In the latter group, GSH levels were similar to those of the healthy, untreated control mice $(1.3 \pm 0.4 \mathrm{mg} / \mathrm{dl}$; Fig. 3 , white bar).

Quercetin does not decrease the severity of pre-neoplastic lesions and OSCC. In the current study, tongues from 46 surviving animals were analyzed. The type and severity of the lesions were determined according to the WHO International Histological Classification of Tumors and Histological Malignancy Grading System for the ITF (as described by Bryne et al) (24).

Mice that were treated with 4-NQO alone and in combination with the different doses of quercetin (10 and $100 \mathrm{mg} / \mathrm{kg} / \mathrm{day}$ ) exhibited various pre-neoplastic lesions, including papilloma, hyperplasia and different degrees of dysplasia, as well as OSCC (Fig. 4A). No statistically significant difference in the relative frequencies of these lesions was identified between the experimental groups $(\mathrm{P}=0.339)$. 

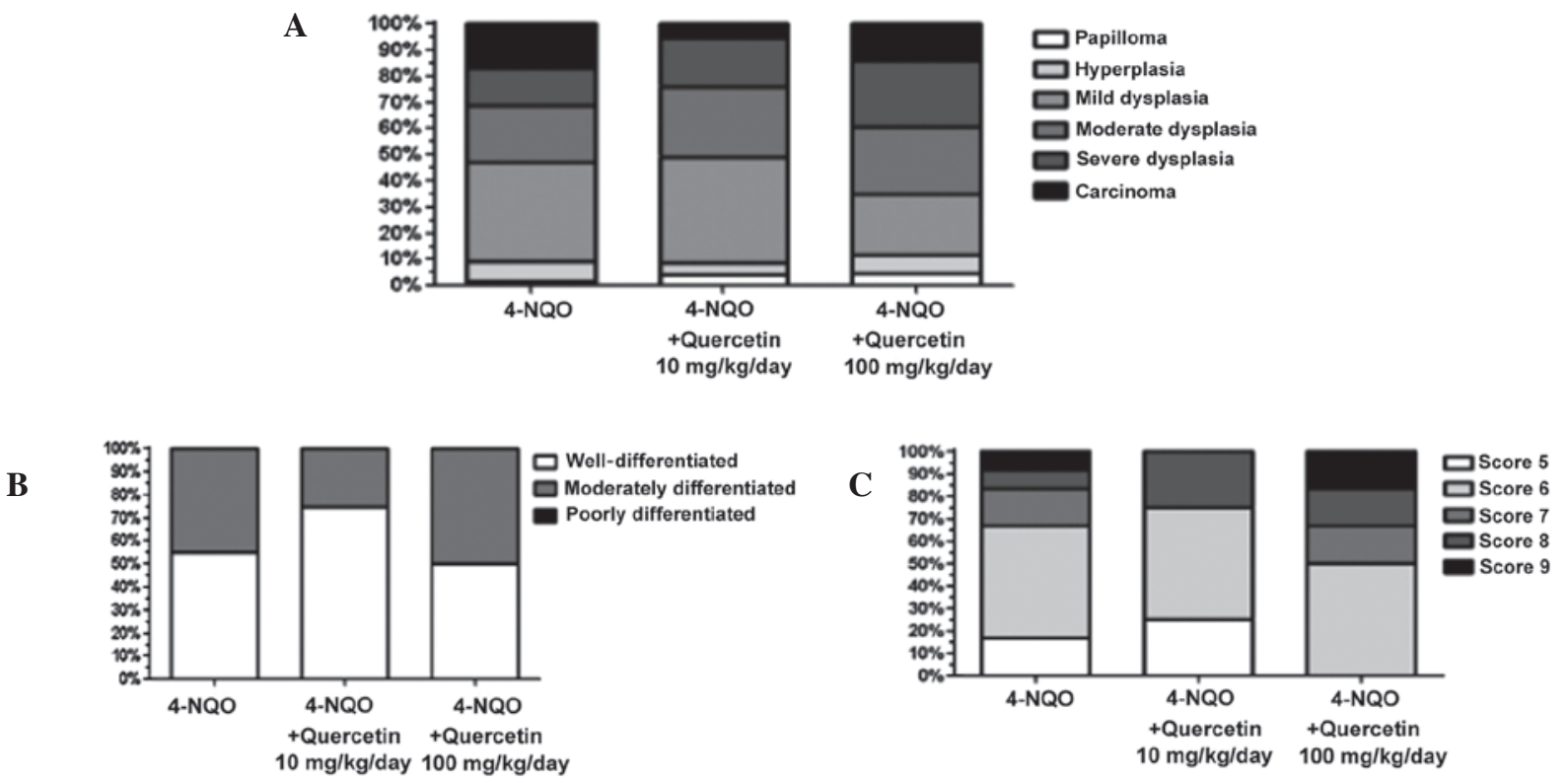

Figure 4. Quercetin does not reduce the severity of pre-neoplastic lesions, OSCC or ITF. Mice were treated with 4-NQO alone or in combination with quercetin $(10 \mathrm{mg} / \mathrm{kg} / \mathrm{day}$ or $100 \mathrm{mg} / \mathrm{kg} /$ day). The tongues of the animals were dissected and processed for routine histopathological analysis (hematoxylin and eosin staining). (A) Lesions were classified as papilloma, hyperplasia, mild dysplasia, moderate dysplasia, severe dysplasia or carcinoma. No statistically significant differences between the groups were observed. Data were analyzed using Fisher's exact test $(\mathrm{P}=0.339)$. (B) A total of 21 lesions were diagnosed as OSCC and classified according to the International Histological Classification of Tumors. None of the analyzed samples was diagnosed as poorly differentiated OSCC. The graph shows the percentage of the classified OSCCs; no statistically significant differences between groups were observed. Data were analyzed using Fisher's exact test ( $\mathrm{P}=0.724)$. (C) OSCC samples were classified according to the Histological Malignancy Grading System for the ITF (20). Scores of the ITF range from 5-9. No statistically significant differences between the groups were observed. Data were analyzed using Fisher's exact test $(\mathrm{P}=1)$. OSCC, oral squamous cell carcinoma; ITF, invasive tumor front; 4-NQO, 4-nitroquinoline 1-oxide.

In healthy, untreated control mice, only healthy mucosa was observed (data not shown).

A total of 21 lesions (Fig. 4B-C) were diagnosed as OSCC and classified using the aforementioned classification systems. None of the samples analyzed was diagnosed as poorly differentiated OSCC. Additionally, there was no statistically significant difference in the relative frequencies of moderately and well-differentiated lesions between animals treated with 4-NQO alone or with the carcinogen plus quercetin $(\mathrm{P}=0.724$, Fig. 4B), despite a higher percentage of well-differentiated lesions in animals treated with the lower dose of quercetin.

The OSCC scores ranged from 5-9 in the Histological Malignancy Grading System for the ITF; these numbers are considered standard for well- and moderately differentiated lesions. Qualitative analysis and Fisher's exact test, performed using the mean of the data, revealed no statistically significant difference $(\mathrm{P}=1)$ between animals treated with 4-NQO alone or with the carcinogen plus quercetin (Fig. 4C).

A histochemical analysis of the ECM in peritumoral tissue was also performed. As expected, OSCCs, independently of the treatment used, exhibited a lower PAS reactivity (Fig. 5B and $\mathrm{C}$ ), particularly in the basal lamina, and a marked disorganization of collagen I (Fig. 5E and F) relative to healthy control mucosa (Fig. 5A and D). However, there were no statistically significant differences $[\mathrm{P}=0.054$ (PAS) and $\mathrm{P}=0.346$ (Picro Sirius Red)] between animals treated with 4-NQO alone or with the carcinogen plus quercetin (Fig. 5C and F).

Treatment with low-dose quercetin decreases the immunoreactivity of tumor markers. OSCCs were analyzed for the presence of the proliferation marker PCNA, which increases in proliferating cells and is involved in the induction of DNA repair at the S phase cell cycle checkpoint (26). OSCCs from animals treated with the lower dose of quercetin $(10 \mathrm{mg} / \mathrm{kg} /$ day $)$ displayed significantly reduced PCNA immunoreactivity compared with animals that had received 4-NQO alone or the carcinogen plus the higher dose of quercetin (Fig. 5I; $\mathrm{P} \leq 0.001)$. PCNA immunoreactivity was limited to the basal layer of the tongue epithelia in the healthy mucosa (Fig. 5G, arrows) whilst, in OSCC tissues, the proliferation marker was observable throughout the entire lesion (Fig. 5H, arrows).

Another important tumor marker is the mutant variant of p53, a tumor suppressor protein that is normally expressed in the presence of DNA damage (27). Immunohistochemical analysis of mutated p53 in OSCC revealed increased immunoreactivity in all groups treated with 4-NQO, including with and without quercetin (Fig. 5J), compared with that of healthy tongue mucosa, where the expression of this protein was absent (Fig. 5K). OSCCs from animals that received the lower dose of quercetin exhibited significantly less intense immunoreactivity of p53 compared with those treated with the carcinogen alone or with the carcinogen plus the higher dose of quercetin $(\mathrm{P} \leq 0.05$; Fig. 5L).

\section{Discussion}

The high incidence and mortality of OSCC, as well as the limited treatment modalities currently available for this cancer, increases the urgency to develop novel therapies for these patients (1). Cancer chemoprevention, defined as long-term intervention with natural or synthetic molecules to prevent, inhibit or reverse carcinogenesis, is increasing in importance, 
A
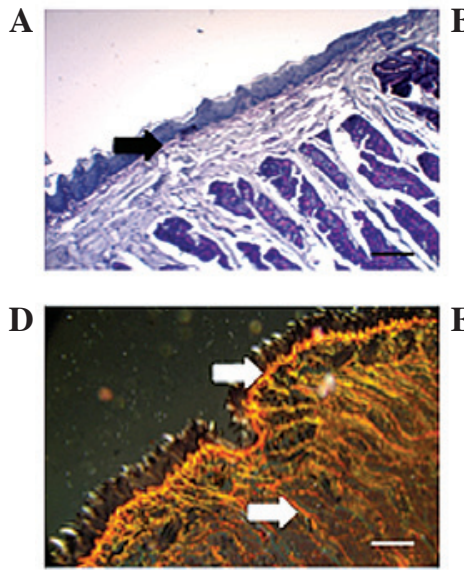

G
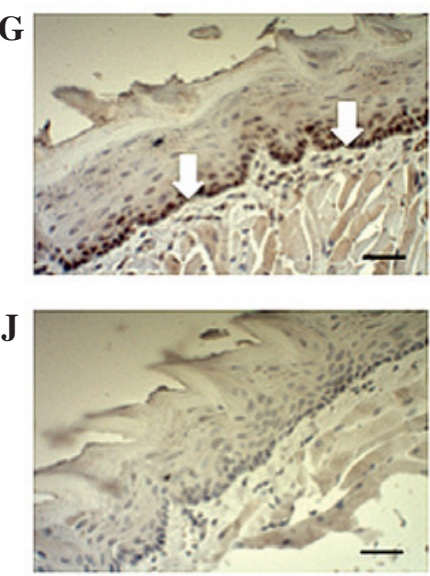
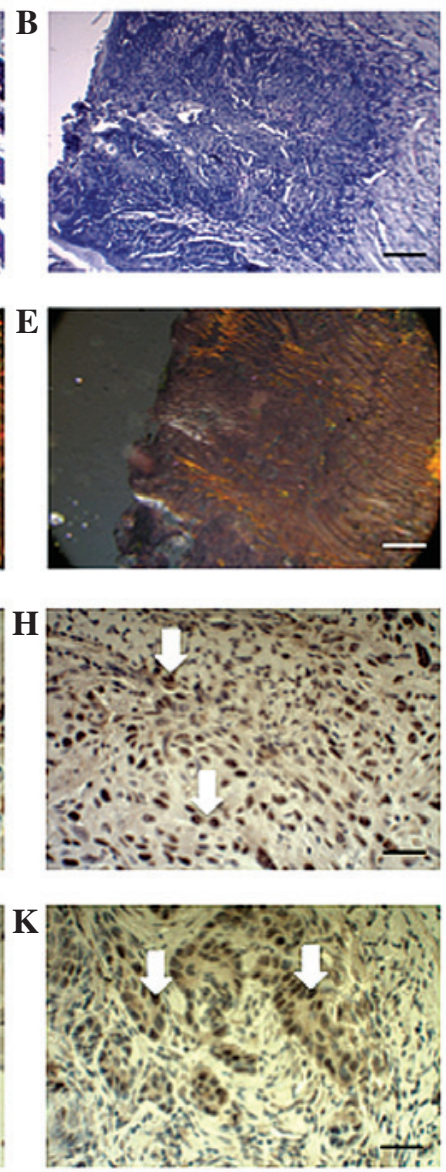

C

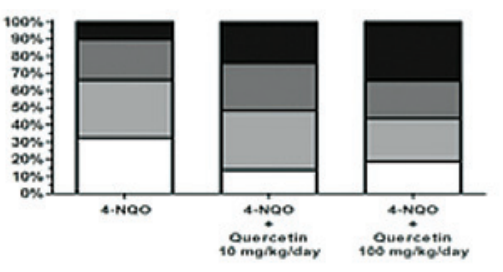

F

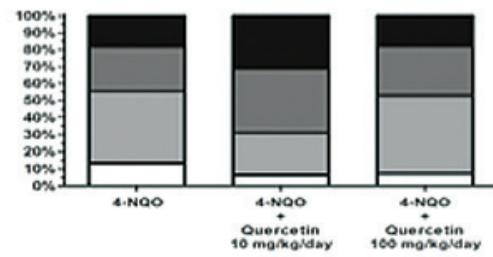

I

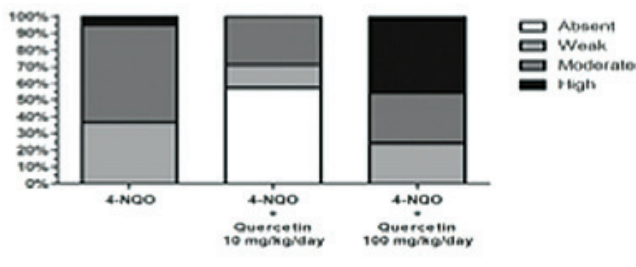

L

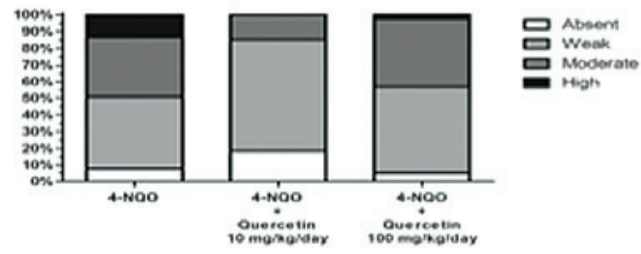

Figure 5. Quercetin does not ameliorate changes in the peritumoral extracellular matrix of OSCC; however, treatment with quercetin at a low dose decreases the immunoreactivity of tumor markers. Mice were treated with 4-NQO alone or in combination with $10 \mathrm{mg} / \mathrm{kg} / \mathrm{day}$ or $100 \mathrm{mg} / \mathrm{kg} / \mathrm{day}$ quercetin. OSCCs and healthy mucosa samples were processed for routine histochemistry and stained with (A-C) PAS to detect glycosylated components, or with (D-F) Picro Sirius Red for collagen histochemistry (scale bar, $25 \mu \mathrm{m})$. Additionally, processed samples were immunohistochemically stained for (G-I) PCNA, or (J-L) mutated p53, and counterstained with Mayer's hematoxylin (scale bar, $25 \mu \mathrm{m}$ ). (A, D, G and J) Representative images from healthy oral mucosa; (B, E, H and K) representative images from moderately differentiated OSCC treated with 4-NQO alone. In healthy tissue, (A) strong PAS reactivity is evident, particularly at the basal lamina (black arrow) and (D) strong collagen I staining can be observed (white arrows). In OSCCs, the (B) PAS and (E) collagen I reactivity observed is notably decreased. PCNA immunoreactivity in (G) healthy mucosa is limited to the basal epithelial layer (white arrows), while in (H) OSCC tissue, high immunoreactivity is evident throughout the entire tumor (white arrows). (J) No immunoreactivity for mutated p53 is evident in healthy tissue; however, in (K) OSCC tissue, p53 immunoreactivity is increased and evident throughout the entire tumor. Graphs indicate the analysis of the intensity of histochemical or immunohistochemical reactions from the different experimental conditions: (C) PAS staining, (F) Picro Sirius Red staining (collagen), (I) PCNA staining, and (L) p53 staining. No statistically significant differences in the PAS and Picro Sirius Red histochemical analyses were observed between the groups: Data were analyzed using Fisher's exact test $(\mathrm{P}=0.064$ and $\mathrm{P}=0.346$ for PAS and Picro Sirius Red histochemistry, respectively). Analysis of immunohistochemistry revealed that animals treated with 4-NQO plus the low dose of quercetin exhibited a statistically significant decrease in the immunoreactivity of PCNA and p53 tumor markers, relative to the other experimental conditions $(\mathrm{P}<0.001)$. OSCC, oral squamous cell carcinoma; 4-NQO, 4-nitroquinoline 1-oxide; PAS, periodic acid-Schiff; PCNA, proliferating cell nuclear antigen.

with a number of clinical trails conducted thus far (28) and may provide complementary therapeutic strategies. The flavonoid quercetin is considered to be the prototypical naturally occurring chemopreventive agent, as its biological activities (anti-proliferative, anti-inflammatory, antioxidant, proapoptotic and anti-angiogenic) may act at various stages of carcinogenesis, from initiation to invasion and metastasis. Additionally, this molecule may affect various genetic, biochemical and immunological factors that underlie the development and maintenance of tumors (10).

There are a number of promising studies regarding the anticancer effect of quercetin in oral carcinoma cell lines (29) and animal models $(16,30,31)$. However, studies of in vivo models of different types of OSCCs are controversial (15).

The use of 4-NQO is a valuable technique for inducing OSCC, and induces carcinogenesis in animal models in a manner similar to the natural progression of OSCC in humans $(17,18)$. 4-NQO is a water-soluble quinoline derivative and is known to form DNA adducts. Additionally, it is able to induce oxidative DNA damage and chromosomal breakage (32).

During carcinogenesis, cells acquire various properties that have been designated as the 'hallmarks of cancer' (33). The acquisition of these properties is a consequence of changes in biochemical signal transduction pathways resulting from the activation of oncogenes and the inactivation of tumor suppressor genes (33). It has been hypothesized that quercetin is able to interfere with different aspects of the 'hallmarks of cancer', and this drug has therefore been proposed to be a potential multi-target inhibitor with pleiotropic and synergistic effects in tumor cells (10).

The cancer-preventive effects of quercetin have been attributed primarily to its antioxidant activity, a property 
that is facilitated by its chemical structure. Quercetin contains a high number of hydroxyl groups and conjugated $\pi$ orbitals, by which this flavonoid can donate electrons or hydrogen, as well as scavenge hydrogen peroxide and superoxide anions. The reaction of quercetin with superoxide anions leads to the generation of semiquinone radicals and hydrogen peroxide. Quercetin also reacts with hydrogen peroxide in the presence of peroxidases, thus decreasing hydrogen peroxide levels and protecting cells against oxidative damage (28). However, in the present study, quercetin was not found to exert significant positive effects against 4-NQO-induced OSCC in mice. Mice treated with the carcinogen alone or in combination with either dose of quercetin exhibited similar mortality rates and severity of pre-neoplastic lesions and OSCC. It is important to note that quercetin, as a potent antioxidant, becomes oxidized to generate quercetin-quinone with its tautomeric forms. Similarly to other semiquinone radicals and quinones, quercetin-quinone is toxic due to its ability to arylate protein thiols (34). Protection against quercetin-quinone may be provided by GSH, which forms transient adducts (6-glutathionyl-quercetin) that possess an extremely short half-life and which rapidly dissociate into GSH and quercetin-quinone (10). This observation suggests that, with a low GSH concentration, quercetin-quinone trapping may be inefficient, and quercetin-quinones may therefore freely react with other thiol groups, such as protein sulfhydryls (10), or DNA. GSH levels are decreased following prolonged exposure to quercetin, suggesting an inability of quercetin to cope with ROS for extended periods. As a consequence, the pro-oxidant effect of quercetin may be greater than its antioxidant effect (28). In the current study, the administration of quercetin over 16 weeks was confirmed to decrease plasmatic levels of GSH in a dose-dependent manner. Notably, the decrease in GSH was observed 18 weeks after final administration of quercetin; therefore, it is more probable that quercetin acts as a pro-oxidant in the current model of experimental carcinogenesis.

However, the antioxidant activity of quercetin is not its only mechanism of action; quercetin also interacts with different proteins (e.g. Bcl-2 proteins and caspases), directly or indirectly, to inhibit survival signaling cascades, including phosphoinositide 3-kinase/Akt, mitogen-activated protein kinases and protein kinase $\mathrm{C}$ pathways. This promotes the release of cytochrome $c$ and the activation of caspases, thereby triggering apoptotic cell death. Additionally, quercetin is able to interact with cell cycle regulatory proteins and trigger $\mathrm{G} 2 / \mathrm{M}$ phase cell cycle arrest in vitro; in human cervical cancer (HeLa) cells, this effect appears to be mediated through the activation of p53 (13). In the present study, the proliferation markers PCNA and p53 displayed significantly decreased immunoreactivity in the OSCC tissues of mice treated with the lower dose of quercetin.

Another proposed mechanism of action of quercetin involves the entry of the flavonoid into epithelial cells and its concentration in the mitochondria and nucleus. In the cytosol, quercetin disrupts the actin cytoskeleton and inhibits cellular proliferation and migration. In the nucleus, the transcription of various genes associated with different cellular processes may be modified by quercetin; such processes include cell motility, cell cycle regulation, xenobiotic metabolism, immune-related factors and transcription (35). However, as mentioned previously, only the immunoreactivity of PCNA and p53 was diminished, and Histological Malignancy Grading System for the ITF scores and changes in tumor-adjacent ECM were not improved in quercetin-treated mice compared with 4-NQO-only-treated mice. This result may indirectly indicate that cell motility and invasion capacity were not affected by quercetin.

An additional explanation of the failure of quercetin in the current model may be related to its bioavailability. Many phytochemicals are poorly absorbed, and the unabsorbed fraction typically undergoes metabolism and rapid excretion (36). Quercetin molecules are differentially glycosylated in food sources, and the adsorption of quercetin glycosides is almost double that of its corresponding aglycon (10), which was administered in the present study. However, as quercetin induced a dose-dependent decrease of plasmatic GSH levels in this study (Fig. 3), it may be assumed that the animals absorbed a sufficient quantity of the flavonoid.

The current results suggest that, despite a promising effect of the flavonoid reported in previous studies (in vitro or in vivo), quercetin, at the doses assayed, is ineffective as a chemopreventive agent. However, interpretation of these results must take account of the fact that 4-NQO does not induce poorly differentiated OSCC. Therefore, considering the effect of the low dose of this flavonoid on tumor marker expression, it is important to investigate the effect of quercetin at lower doses, as well as on more severe lesions.

\section{Acknowledgements}

This study was supported by National Fund for Scientific and Technological Development grants no. 1120230 (to Dr Ulrike Kemmerling), no. 1120096 (to Dr Guillermo Schmeda-Hirschmann), and no. 1110054 (to Mrs. Cristina Theoduloz), and by the 'Fondo para la Realización de Tesis del Programa de Magister en Ciencias Biomédicas de la Facultad de Ciencias de la Salud de la Universidad de Talca'.

\section{References}

1. Bai LY, Weng JR, Hu JL, Wang D, Sargeant AM and Chiu CF: G15, a GPR30 antagonist, induces apoptosis and autophagy in human oral squamous carcinoma cells. Chem Biol Interact 206: 375-384, 2013.

2. Liu Y, Zha L, Li B, Zhang L, Yu T and Li L: Correlation between superoxide dismutase 1 and 2 polymorphisms and susceptibility to oral squamous cell carcinoma. Exp Ther Med 7: 171-178, 2014.

3. Lin WJ, Jiang RS, Wu SH, Chen FJ and Liu SA: Smoking, alcohol and betel quid and oral cancer: A prospective cohort study. J Oncol 2011: 525976, 2011.

4. Hollows P, McAndrew PG and Perini MG: Delays in the referral and treatment of oral squamous cell carcinoma. Br Dent J 188: 262-265, 2000.

5. Taghavi $\mathrm{N}$ and Yazdi I: Prognostic factors of survival rate in oral squamous cell carcinoma: Clinical, histologic, genetic and molecular concepts. Arch Iran Med 18: 314-319, 2015.

6. Vermorken JB, Remenar E, van Herpen C, Gorlia T, Mesia R, Degardin M, Stewart JS, Jelic S, Betka J, Preiss JH, et al: Cisplatin, fluorouracil and docetaxel in unresectable head and neck cancer. N Engl J Med 357: 1695-1704, 2007.

7. Ames BN and Gold LS: Endogenous mutagens and the causes of aging and cancer. Mutat Res 250: 3-16, 1991

8. Korde SD, Basak A, Chaudhary M, Goyal M and Vagga A: Enhanced nitrosative and oxidative stress with decreased total antioxidant capacity in patients with oral precancer and oral squamous cell carcinoma. Oncology 80: 382-389, 2011.

9. Dolatabadi JE: Molecular aspects on the interaction of quercetin and its metal complexes with DNA. Int J Biol Macromol 48: 227-233, 2011. 
10. Russo M, Spagnuolo C, Tedesco I, Bilotto S and Russo GL: The flavonoid quercetin in disease prevention and therapy: Facts and fancies. Biochem Pharmacol 83: 6-15, 2012.

11. Borska S, Chmielewska M, Wysocka T, Drag-Zalesinska M, Zabel $\mathrm{M}$ and Dziegiel P: In vitro effect of quercetin on human gastric carcinoma: Targeting cancer cells death and MDR. Food Chem Toxicol 50: 3375-3383, 2012.

12. Kang JW, Kim JH, Song K, Kim SH, Yoon JH and Kim KS: Kaempferol and quercetin, components of Ginkgo biloba extract (EGb 761), induce caspase-3-dependent apoptosis in oral cavity cancer cells. Phytother Res 24 (Suppl 1): S77-S82, 2010.

13. Dajas F: Life or death: Neuroprotective and anticancer effects of quercetin. J Ethnopharmacol 143: 383-396, 2012.

14. Murakami A, Ashida $\mathrm{H}$ and Terao J: Multitargeted cancer prevention by quercetin. Cancer Lett 269: 315-325, 2008.

15. Harwood M, Danielewska-Nikiel B, Borzelleca JF, Flamm GW Williams GM and Lines TC: A critical review of the data related to the safety of quercetin and lack of evidence of in vivo toxicity, including lack of genotoxic/carcinogenic properties. Food Chem Toxicol 45: 2179-2205, 2007.

16. Yang CS, Landau JM, Huang MT and Newmark HL: Inhibition of carcinogenesis by dietary polyphenolic compounds. Annu Rev Nutr 21: 381-406, 2001.

17. Dayan D, Hirshberg A, Kaplan I, Rotem N and Bodner L: Experimental tongue cancer in desalivated rats. Oral Oncol 33: 105-109, 1997.

18. Rivera CA, Droguett DA, Kemmerling U and Venegas BA: Chronic restraint stress in oral squamous cell carcinoma. J Dent Res 90: 799-803, 2011

19. Tang XH, Knudsen B, Bemis D, Tickoo S and Gudas LJ: Oral cavity and esophageal carcinogenesis modeled in carcinogen-treated mice. Clin Cancer Res 10: 301-313, 2004.

20. Junqueira LC, Bignolas G and Brentani RR: Picrosirius staining plus polarization microscopy, a specific method for collagen detection in tissue sections. Histochem J 11: 447-455, 1979.

21. Castillo C, López-Muñoz R, Duaso J, Galanti N, Jaña F, Ferreira J, Cabrera G, Maya JD and Kemmerling U: Role of matrix metalloproteinases 2 and 9 in ex vivo Trypanosoma cruzi infection of human placental chorionic villi. Placenta 33: 991-997, 2012.

22. Pindborg JJ, Reichert PA, Smith CJ and van de Waal I (eds): Histological typing of cancer and precancer of the oral mucosa. In: World Health Organization Histological Classification of Tumours 2nd edition. Springer, Berlin, pp11-12, 1997.

23. Tumuluri V, Thomas GA and Fraser IS: Analysis of the Ki-67 antigen at the invasive tumour front of human oral squamous cell carcinoma. J Oral Pathol Med 31: 598-604, 2002.

24. Bryne M, Boysen M, Alfsen CG, Abeler VM, Sudbø J, Nesland JM, Kristensen GB, Piffko J and Bankfalvi A: The invasive front of carcinomas. The most important area for tumour prognosis? Anticancer Res 18: 4757-4764, 1998.
25. Beutler E, Duron O and Kelly BM: Improved method for the determination of blood glutathione. J Lab Clin Med 61: 882-888, 1963.

26. Myoung H, Kim MJ, Lee JH, Ok YJ, Paeng JY and Yun PY: Correlation of proliferative markers (Ki-67 and PCNA) with survival and lymph node metastasis in oral squamous cell carcinoma: A clinical and histopathological analysis of 113 patients. Int J Oral Maxillofac Surg 35: 1005-1010, 2006.

27. Li L, Fukumoto M and Liu D: Prognostic significance of p53 immunoexpression in the survival of oral squamous cell carcinoma patients treated with surgery and neoadjuvant chemotherapy. Oncol Lett 6: 1611-1615, 2013.

28. Gibellini L, Pinti M, Nasi M, Montagna JP, De Biasi S, Roat E, Bertoncelli L, Cooper EL and Cossarizza A: Quercetin and cancer chemoprevention. Evid Based Complement Alternat Med 2011: 591356, 2011.

29. Chen SF, Nien S, Wu CH, Liu CL, Chang YC and Lin YS: Reappraisal of the anticancer efficacy of quercetin in oral cancer cells. J Chin Med Assoc 76: 146-152, 2013.

30. Makita H, Tanaka T, Fujitsuka H, Tatematsu N, Satoh K, Hara A and Mori H: Chemoprevention of 4-nitroquinoline 1-oxide-induced rat oral carcinogenesis by the dietary flavonoids chalcone, 2-hydroxychalcone and quercetin. Cancer Res 56: 4904-4909, 1996.

31. Priyadarsini RV, Vinothini G, Murugan RS, Manikandan $P$ and Nagini S: The flavonoid quercetin modulates the hallmark capabilities of hamster buccal pouch tumors. Nutr Cancer 63: 218-226, 2011.

32. Ribeiro FA, de Moura CF, Gollucke AP, Ferreira MS, Catharino RR, Aguiar O Jr, Spadari RC, Barbisan LF and Ribeiro DA: Chemopreventive activity of apple extract following medium-term oral carcinogenesis assay induced by 4-nitroquinoline-1-oxide. Arch Oral Biol 59: 815-821, 2014.

33. Hanahan D and Weinberg RA: Hallmarks of cancer: The next generation. Cell 144: 646-674, 2011.

34. Gliszczyńska-Świglo A and Oszmianśki J: Antioxidant and prooxidant activity of food components. In: Food Oxidants and Antioxidants: Chemical, Biological, and Functional Properties. Bartosz G (ed). CRC Press, Boca Raton, FL, pp375-432, 2013.

35. Notas G, Nifli AP, Kampa M, Pelekanou V, Alexaki VI, Theodoropoulos P, Vercauteren J and Castanas E: Quercetin accumulates in nuclear structures and triggers specific gene expression in epithelial cells. J Nutr Biochem 23: 656-666, 2012.

36. Johnson IT: Phytochemicals and cancer. Proc Nutr Soc 66: 207-215, 2007 